\title{
NUTRIGENÓMICA Y NUTRIGENÉTICA: EL FUTURO DE LA NUTRICIÓN
}

\section{Nutrigenomics and Nutrigenetics The future of nutrition}

Recibido: 28-05-19

Aceptado: 05-06-19

\section{Por: MSc. Mariel Montealegre Basadre}

Nutricionista.

\section{Introducción}

Las guías de nutrición para anticipar padecimientos metabólicos establecen sugerencias generales sobre macronutrientes y micronutrientes. Están sugerencias no son para toda la población, por tal razón es imprescindible estar al tanto de la nutrigenómica y la nutrigenética de cada individuo

Palabras claves :nutrigenómica, nutrigenética, patología.

\section{Abstract}

Nutrition guidelines for anticipating metabolic disorders establish general suggestions on macronutrients and micronutrients. These suggestions are not for the entire population, for this reason it is essential to be aware of the nutrigenomics and nutrigenetics of each individual

Keywords: nutrigenomics, nutrigenetics, pathology

Actualmente las guías de nutrición para prevenir las diversas enfermedades metabólicas se

basan en recomendaciones generales sobre macronutrientes y micronutrientes. No obstante, las recomendaciones generalizadas no son las indicadas para toda la población, ya que existen individuos que presentan variantes genéticas las cuales aumentan o disminuyen sus requerimientos de ciertos nutrientes, vitaminas y minerales. Debido a esto, es importante conocer acerca de la nutrigenómica y la nutrigenética, con el fin de poder fomentar una nutrición más personalizada y por lo tanto efectiva (Bashiardes, Godneva, Elinav, \& Segal, 2018; Peña, Navas,
Marín, \& Orenes, 2017).

La nutrición personalizada nace gracias a la genómica nutricional, la cual se puede dividir en dos principales ramas: la nutrigenómica y la nutrigénetica. La nutrigenómica se puede definir como la ciencia que estudia la influencia de los nutrientes en la expresión génica, mientras que la nutrigenética eslacienciaqueestudiacómodistintas variaciones genéticas afectan las respuestas a la dieta, los requerimientos nutricionales, y hasta las preferencias alimentarias. Ambas han tenido un crecimiento significativo desde el 2003, cuando la determinación del genoma humano permitió el avance de diversas investigaciones, cuyo objetivo 
era aprender sobre las diferentes interacciones entre genes y nutrientes (Peña, Navas, Marín, \& Orenes, 2017).

La Nutrigenética potencia la prevención de patologías, ya que existe la posibilidad de introducir cambios dietéticos desde muy pronto en personas con una mayor predisposición genética a alguna enfermedad. De igual manera, se ha visto que los individuos que conocen su genética podrían estar más motivados a realizar un cambio de estilo de vida.

Por lo tanto, tanto la genética como el ambiente de un individuo participan en el fenotipo del mismo. Aunque sabemos que las personas no pueden cambiar su material genético, sí pueden alimentarse de la mejor manera posible para poder respaldar las predisposiciones con las que éste cuenta. (Aruoma, et al., 2019) De igual forma, la nutrigenómica, permite conocer los numerosos componentes dietéticos y bioactivos que pueden alterar los eventos génicos y epigenéticos, y por lo tanto influenciar la salud (Trujillo, Davis, \& Milner, 2006).

No hay duda que tanto la nutrigenómica como la nutrigénetica son ciencias en constante crecimiento en muchas áreas de investigación, y que serán cada vez más utilizadas por los profesionales en salud con el fin de establecer estrategias de intervención dietéticas más eficientes dirigidas a prevenir enfermedades, mejorar la calidad de vida y lograr un envejecimiento saludable (Peña, Navas, Marín, \& Orenes, 2017).

\section{BIBLIOGRAFÍA}

Aruoma, O., Hausman-Cohen, S., Pizano, J., Schmidt, M., Minich, D., Joffe, Y., . . Brady, D. (2019). Personalized Nutrition: Translating the Science of NutriGenomics Into Practice: Proceedings From the 2018 American College of Nutrition Meeting. Journal of the American College of Nutrition, 287-301.

Bashiardes, S., Godneva, A., Elinav, E., \& Segal, E. (2018). Towards utilization of the human genome and microbiome for personalized nutrition. Current Opinion in Biotechnology, 57-63.

Peña-Romero, A., Navas-Carrillo, D., Marín, F., \& Orenes-Piñero, E. (2017). The future of Nutrition: Nutrigenomics and Nutrigenetics in Obesity and Cardiovascular Diseases. Food Science and Nutrition, 1-12.

Trujillo, E., Davis, C., \& Milner, J. (2006). Nutrigenomics, Proteomics, Metabolomics, and the Practice of Dietetics. ournal of the AMERICAN DIETETIC ASSOCIATION, 403-413. 